\title{
Single cell transfection by laser-induced breakdown of an optically trapped gold nanoparticle
}

\author{
Yoshihiko Arita $^{a}$, Martin Ploschner ${ }^{a}$, Maciej Antkowiak ${ }^{a, b}$, Frank Gunn-Moore ${ }^{b}$ and Kishan \\ Dholakia $^{a}$ \\ ${ }^{a}$ SUPA, School of Physics and Astronomy, University of St Andrews, St Andrews, Fife, KY16 \\ 9SS, United Kingdom; \\ ${ }^{b}$ SULSA, School of Biology, Medical and Biological Sciences Building, University of St \\ Andrews, St Andrews, Fife, KY16 9TF, United Kingdom
}

\begin{abstract}
Cell selective introduction of therapeutic agents remains a challenging problem. Cavitation-based therapies including ultrasound-induced sonoporation and laser-induced optoporation have led the way for novel approaches to provide the potential of sterility and cell selectivity compared with viral or biochemical counterparts. Acoustic streaming, shockwaves and liquid microjets associated with the cavitation dynamics are implicated in gene and drug delivery. These approaches, however, often lead to non-uniform and sporadic molecular uptake that lacks refined spatial control and suffers from a significant loss of cell viability. Here we demonstrate spatially controlled cavitation instigated by laser-induced breakdown of an optically trapped single gold nanoparticle. Our unique approach employs optical tweezers to trap a single nanoparticle, which when irradiated by a nanosecond laser pulse is subject to laser-induced breakdown followed by cavitation. Using this method for laser-induced cavitation, we can gain additional degrees of freedom for the cavitation process - the particle material, its size, and its position relative to cells or tissues. We show the energy breakdown threshold of gold nanoparticles of $100 \mathrm{~nm}$ with a single nanosecond laser pulse at $532 \mathrm{~nm}$ is three orders of magnitude lower than that for water, which leads to gentle nanocavitation enabling single cell transfection. We optimize the shear stress to the cells from the expanding bubble to be in the range of $1-10 \mathrm{kPa}$ for transfection by precisely positioning a trapped gold nanoparticle, and thus nanobubble, relative to a cell of interest. The method shows transfection of plasmid-DNA into individual mammalian cells with an efficiency of $75 \%$.
\end{abstract}

Keywords: Laser-induced breakdown, Laser trapping, Ultrasound, Surface plasmons

\section{INTRODUCTION}

The transfection of genes and injection of therapeutic agents into individual mammalian cells are among the most important research tools in modern molecular biology. ${ }^{1}$ The use of acoustic bubbles in the proximity of cells oscillated by ultrasonic irradiation (insonation) can lead to enhanced membrane permeabilization of cells, and is known as sonoporation. Acoustic streaming, shock waves, and liquid microjets associated with the dynamics of cavitation bubble are implicated in gene and drug delivery into cells. ${ }^{2}$ This approach, however, often leads to nonuniform and sporadic molecular uptake that lacks cell selectivity and suffers from a significant loss of cell viability.

Recently a suite of optical methods in the domain of cavitation-based therapies has provided the potential of sterility, reconfigurability, and single cell selectivity. ${ }^{3}$ Laser-induced breakdown (LIB) of a liquid medium containing cells, has demonstrated cell lysis, necrosis or membrane permeabilization, with the outcome dependent upon the hydrodynamic shear stress to cells caused by the expanding bubble. ${ }^{4}$ However, the relatively high energy deposition required for this process resulted in a much larger cavitation bubble (typically $>200 \mu \mathrm{m}$ in diameter) compared to the typical cell size that effectively reduces cell viability and has been detrimental to allow its wider usage.

Further author information: (Send correspondence to Y.A.)

Y.A.: E-mail: ya10@st-andrews.ac.uk, Telephone: +44 (0)1334 461656

Frontiers in Ultrafast Optics: Biomedical, Scientific, and Industrial Applications XIV, edited by Alexander Heisterkamp,

Peter R. Herman, Michel Meunier, Stefan Nolte, Proc. of SPIE Vol. 8972, 897203

(C) 2014 SPIE · CCC code: 0277-786X/14/\$18 - doi: 10.1117/12.2039647

Proc. of SPIE Vol. $8972897203-1$ 
More spatially controlled cavitation may be achieved by optically trapping particles for subsequent LIB instead of the surrounding liquid. ${ }^{5}$ Optical tweezers allow the positioning of individual nanoparticles or microparticles at a desired location within the buffer medium. Therefore, using this tool for LIB offers additional degrees of freedom - the particle material, its size, and the LIB position relative to cells or tissues. We have shown that the LIB of trapped polystyrene nanoparticles significantly reduced the energy required for cavitation. ${ }^{6,7}$ This leads to the permeabilization of cell membranes and transfection of cells in a targeted area in the absence of a lysis zone of cells.

In this Letter, we demonstrate LIB of a gold nanoparticle where the energy deposition required for cavitation is three orders of magnitude lower than the breakdown threshold for water. ${ }^{8}$ Manipulation of the nanoparticle position relative to the cell membrane allows exceptional spatial control and targeting of single cells at will for transfection. We model the temperature rise $>10^{6} \mathrm{~K}$ due to heat generation when the trapped gold nanoparticle is irradiated with a single nanosecond laser pulse with a wavelength near the plasmon resonance frequency. We explore the dynamics of the cavitation bubble caused by this localized heat absorption, based on the nanosecond time-resolved images of cavitation. This allows us to quantify the hydrodynamic shear stress created by the expanding bubble at different radial positions, at different times. We demonstrate transfection of plasmid-DNA into CHO-K1 cells with an efficiency of $75 \%$ by optimizing the shear stress on cells, i.e., optimizing the relative position between the cell and nanoparticle (and thus nanobubble), without any loss of cell viability. ${ }^{8}$

\section{MATERIALS AND METHODS}

\subsection{Laser trapping and laser-induced breakdown}

A single gold nanoparticle (100 $\mathrm{nm}$ in diameter) was trapped by a $1070 \mathrm{~nm}$ continuous wave (CW) fiber laser (IPG Laser $\mathrm{GmbH}$, power of $80 \mathrm{~mW}$ ). A single pulse of $532 \mathrm{~nm}$ frequency-doubled Q-switched Nd:YAG laser (Elforlight Ltd., pulse width of $1 \mathrm{~ns}$, energy of $10 \mathrm{~nJ}$ ) was used to effect LIB of the trapped gold nanoparticle. Both of the beams were coaligned and focused by the same microscope objective (Nikon Ltd., 100×, NA=1.25 in oil) at a predefined axial location of $10 \mu \mathrm{m}$ above the substrate, but within the cell culture medium. (See Fig. 1.) Quadrant photodiodes were used to monitor the scattered light from the trapped particle to ensure it was solely a single gold nanoparticle that was held in the trap.

\subsection{Time-resolved imaging}

A second $532 \mathrm{~nm} \mathrm{Nd:YAG} \mathrm{laser} \mathrm{(Elforlight} \mathrm{Ltd.,} \mathrm{pulse} \mathrm{width} \mathrm{of} 1 \mathrm{~ns}$ ) was used for illumination, which delivers a single nanosecond pulse at a desired time delay after the LIB event. Both the nanosecond lasers (one for LIB and the other for illumination) were triggered at a desired timing using a digital delay generator (Stanford Research Systems Inc., time resolution of 5 ps). Images of the LIB events, at time delays between the laser pulses of $0-500 \mathrm{~ns}$, were recorded by a CCD camera (Imaging Source) triggered synchronously with the nanosecond lasers. As a result, one stroboscopic image was captured for each triggered LIB event. (See Fig. 1.)

\subsection{Nanoparticles and cells}

The gold nanoparticles (BBI solutions) were suspended in Opti-MEM (Invitrogen Ltd.), containing plasmid-DNA (encoding Mito-DsRed; Clontech) of $10 \mu \mathrm{gm} \ell^{-1}$, to yield a concentration of $10^{6} \mathrm{~m} \ell^{-1}$. CHO-K1 cells cultured on a glass-bottomed dish (WPI Ltd., $35 \mathrm{~mm}$ ) with a confluency of $\leq 50 \%$ were prepared in this stock suspension. Ten individual gold nanoparticles per sample, were carefully positioned at the axial location of $10 \mu \mathrm{m}$ from the substrate and at the radial positions of either $<5,10$, or $50 \mu \mathrm{m}$ from the center of cell nucleus. After 10 LIB events, the stock suspension was replaced with cell culture medium, Dulbeccos Modified Eagles Medium (DMEM, Sigma-Aldrich Co. Ltd.) with 10\% fetal calf serum (FCS, Globepharm Ltd.), $20 \mu \mathrm{gm} \ell^{-1}$ streptomycin (Sigma-Aldrich Co. Ltd.) and $20 \mu \mathrm{gm} \ell^{-1}$ penicillin (Sigma-Aldrich Co. Ltd.). The cells were incubated at $37^{\circ} \mathrm{C}$ with $5 \% \mathrm{CO}_{2}$ for $48 \mathrm{~h}$ to express the gene of interest. A minimum of five samples $(N=5)$ were treated for each radial position in the same manner to obtain reliable statistics. 


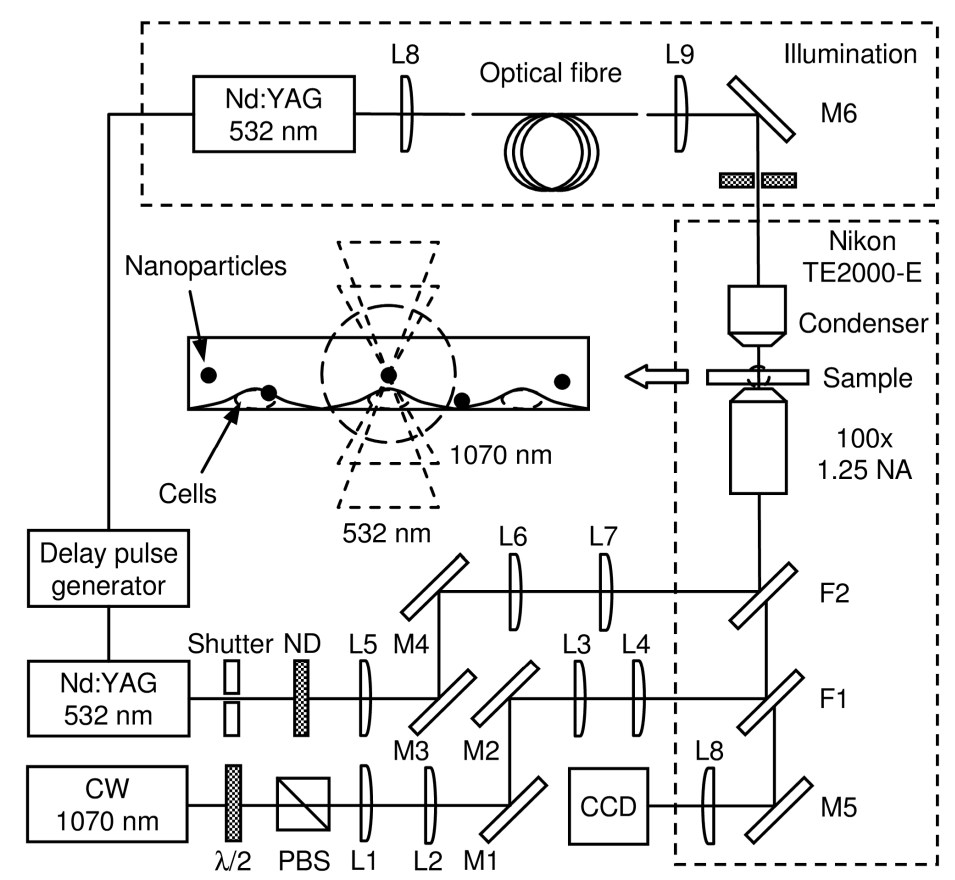

Figure 1. Experimental apparatus for LIB of optically trapped single nanoparticles and nanosecond time-resolved imaging. The $532 \mathrm{~nm}$ Nd:YAGlaser (for LIB) is co-aligned with the $1070 \mathrm{~nm} \mathrm{CW}$ laser (for trapping) through the $100 \times$ oil immersion objective to breakdown the trapped gold nanoparticles (100 $\mathrm{nm}$ in diameter) at a desired location in the sample medium. For imaging, the second $532 \mathrm{~nm} \mathrm{Nd:YAG} \mathrm{laser} \mathrm{is} \mathrm{used} \mathrm{to} \mathrm{deliver} \mathrm{a} \mathrm{single} \mathrm{nanosecondpulse} \mathrm{at} \mathrm{a} \mathrm{desired} \mathrm{time} \mathrm{delay} \mathrm{after}$ the LIB event. Images were recorded by the CCD camera triggered synchronously with the nanosecond lasers. (CW: continuous wave, $\lambda / 2$ : half wave plate, PBS: polarizing beam splitter, ND: neutral density filter, L: lenses, M: mirrors, F1: dichroic filter R80\%@1070nm/T50\%@532nm, F2: dichroic filter R92\%@532nm/T70\%@1070nm)

\section{HEAT TRANSFER MODEL}

We first modeled the amount of energy absorbed by the gold nanoparticle positioned at the focus of the beam (532 nm) using the COMSOL Multiphysics software (RF module in scattering formulation). The incoming beam was considered as a paraxial Gaussian beam in the far-field expression $\mathrm{E}_{\infty}$ of the angular spectrum decomposition, which is a valid approximation when the beam diameter is large $(5 \mathrm{~mm})$ in the back-focal plane of the objective. ${ }^{9}$ We subsequently discretized the far-field reference sphere into 12 intervals in polar angle $\left(0 \ldots 70^{\circ}\right)$, where the maximum polar angle is given by the maximum achievable angle for the objective with $\mathrm{NA}=1.25$ with the interface of glass $\left(n_{g}=1.518\right)$ and water $\left(n_{w}=1.33\right)$, and 30 intervals in azimuthal angle $\left(0 \ldots 360^{\circ}\right)$. This allowed us to model the inside of the far-field reference sphere with the refractive index of water $\left(n_{w}=1.33\right)$ while the outside of the sphere has the index of air $\left(n_{a}=1\right)$. The focal field using these assumptions, which neglect the change in the angular spectrum due to the presence of the glass-water interface, is subsequently utilized for comparison of the power flow in the computational domain either with or without a gold nanoparticle positioned at the highest intensity region of the focal field. This comparison revealed that approximately $38 \%$ of the injected power is absorbed by the gold nanoparticle. This amount of absorption is in line with other work on heating of trapped gold nanoparticles, which uses the same approximations we make. ${ }^{10}$ For a single 1 ns laser pulse, of energy $10 \mathrm{~nJ}$, the heat transfer model shows that the absorbed energy is strongly localized in the close proximity of the gold nanoparticle at the end of the pulse [Fig. 2(a)]. The dissipation of the heat through conduction into water is negligible due to poor thermal conductivity of water in this time scale. The deposited energy is able to raise the temperature of the gold nanoparticle by a million Kelvin [Fig. 2(b)] at the end of the 1 ns pulse. This calculated temperature indicates an upper limit as we neglect the vaporization of water and the phase change of gold. Optical breakdown can take place, leading to the formation of plasma and emission of shockwaves by its expansion followed by the vaporization of the nanoparticle or liquid (surrounding aqueous medium). ${ }^{11}$ 

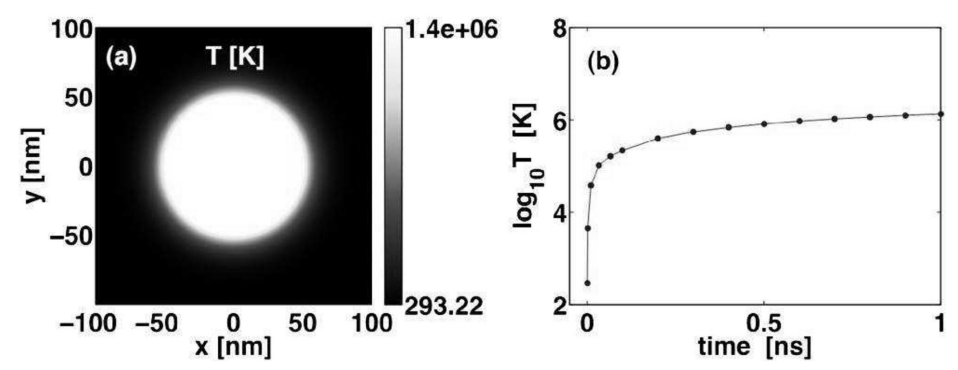

Figure 2. (a) Temperature profile in the vicinity of gold nanoparticle at the end of 1 ns pulse. The absorbed energy from the pulse was modeled as a uniform heat source within the volume of the nanoparticle. (b) Calculated temperature at the surface of the nanoparticle. This increases dramatically in the duration of the pulse.

\section{RESULTS AND DISCUSSIONS}

\subsection{Cavitation dynamics}

Figure 3 shows a series of time-resolved images of the expansion and collapse of the cavitation bubble initiated at an axial position, $Z_{0}$ of $10 \mu \mathrm{m}$ from the substrate. The maximum bubble radius, $R_{\max }$, reached $3 \mu \mathrm{m}$ at $150 \mathrm{~ns}$, which is typically two orders of magnitude smaller than that of the LIB of a liquid alone. A standoff parameter, $\gamma>3=\left(Z_{0} / R_{\max }\right)$ suggests that a jet flow formed towards the nearby substrate during the collapse phase had a minor effect on the experimental outcomes we observed.
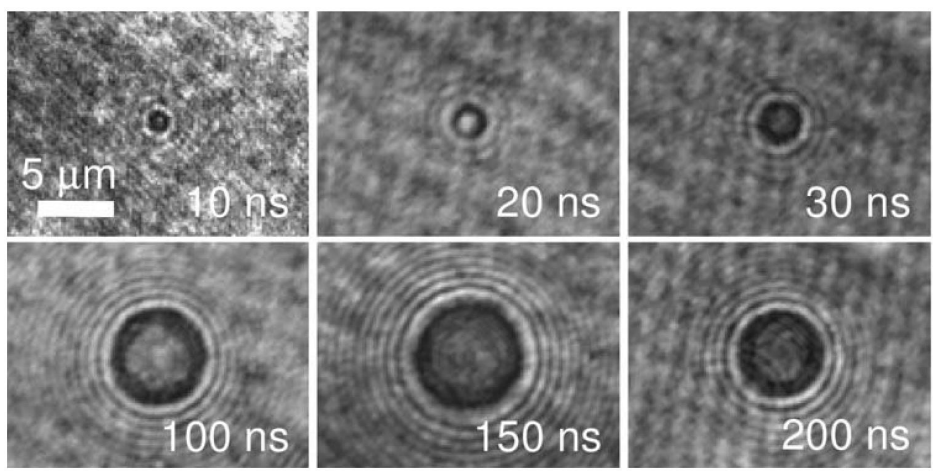

Figure 3. Time-resolved images of cavitation bubbles at time delays between $10 \mathrm{~ns}$ and $200 \mathrm{~ns}$ after the LIB event.

The temporal evolution of the radial position, $R_{B}(t)$, of the bubble wall is shown in Fig. 4(a). A minimum of 10 images were used to obtain the average and standard deviation $(2 \sigma)$ at each data point shown. The bubble wall velocity, $V_{B}(t)=\left(d R_{B}(t) / d t\right)$, is shown in Fig. 4(b). The transient external fluid velocity, $V_{\infty}(r, t)$, produced by the cavitation bubble expansion was determined by applying the conservation of mass for an incompressible fluid in spherical coordinates:

$$
V_{\infty}(r, t)=V_{B}(t)\left[\frac{R_{B}(t)}{r}\right]^{2}
$$

Conservation of momentum was then applied to obtain the transient wall shear stress $\tau_{w}(r, t)$ :

$$
\tau_{w}(r, t)=\rho \sqrt{\frac{\nu}{\pi}} \int_{0}^{t} \frac{\partial V_{\infty}\left(r, t^{\prime}\right)}{\partial t^{\prime}} \frac{d t^{\prime}}{\sqrt{t-t^{\prime}}},
$$

where $\rho\left(=1,000 \mathrm{kgm}^{-3}\right)$ and $\nu\left(=0.896 \times 10^{-6} \mathrm{~m}^{2} \mathrm{~s}^{-1}\right)$ are the density of the liquid and kinematic viscosity of the buffer medium. ${ }^{4,6}$ Equation 2 is valid at any radial position $r$ and time $0<t<t^{\prime}$, where $t^{\prime}$ is the time of arrival 
of the bubble wall at position $r$. The temporal profile of the wall shear stress at different radial positions is shown in Fig. 4(c). The model revealed that the maximum shear stress decreases with increasing radial position, as shown in Fig. 4(d), and is $<1 \mathrm{kPa}$ only at $r=15 \mu \mathrm{m}$, which is typically three orders of magnitude lower than LIB in water alone. ${ }^{4}$
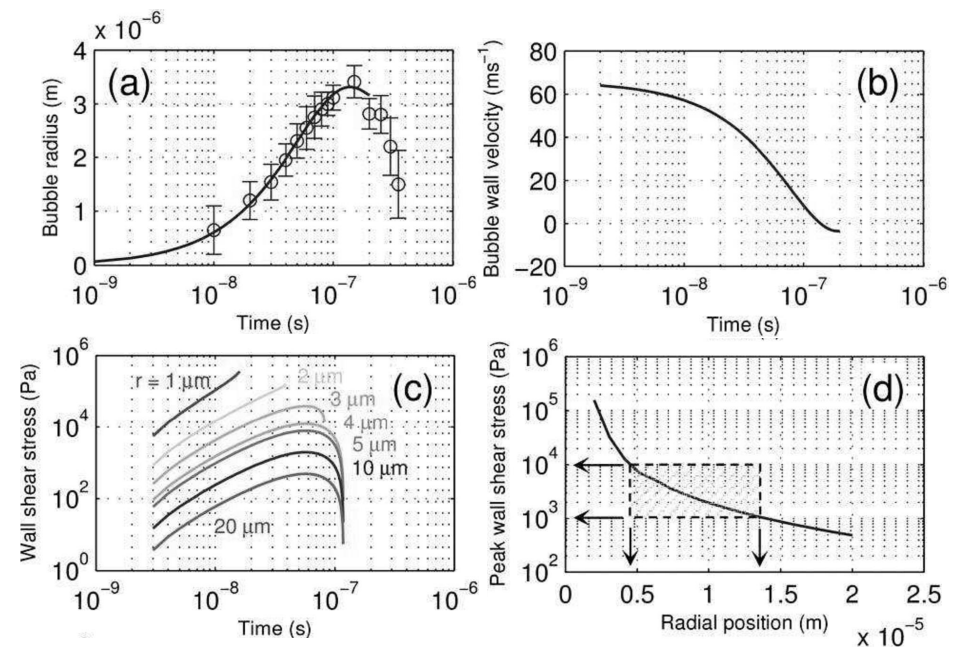

Figure 4. Cavitation bubble dynamics and wall shear stress. (a) Evolution of bubble radius, $R_{B}(t)$. (b) Bubble wall velocity, $V_{B}(t)$. (c) Temporal shear stress, $\tau_{w}(r, t)$ at different radial positions. (d) Maximum shear stress, $\tau_{w, \text { max }}(r)$ at different radial positions.

\subsection{Cell transfection}

We investigated transfection of Mito-DsRed encoding plasmids into CHO-K1 cells at different radial positions. Figure 5(a) shows a phase contrast image of cells and Fig. 5(b) the corresponding fluorescence image of the region of interest ( $48 \mathrm{~h}$ after LIB) showing 11 cells transfected. The number of cells transfected with different radial positions is shown in Fig. 5(c).
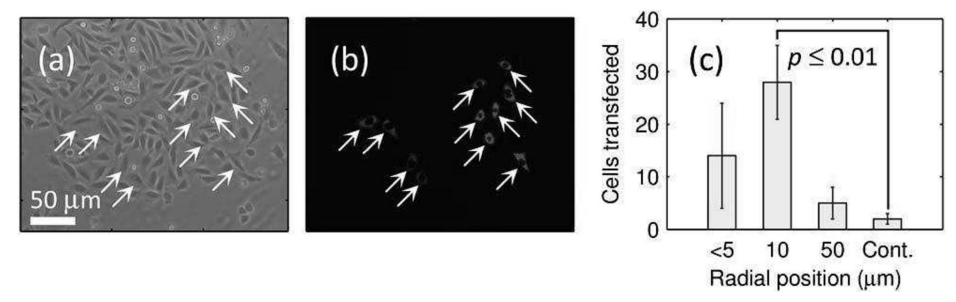

Figure 5. Transfection of Mito-DsRed encoding plasmids into CHO-K1 cells. (a) Phase contrast image of cells in the region of interest. (b) Fluorescence image of (a) showing 11 cells transfected (indicated by arrows). (c) Number of cells transfected with different radial positions ( $48 \mathrm{~h}$ after LIB). The error bars show two standard deviations. $p$ value of $\leq 0.01$ is a result from the unpaired $t$-test between $r=10 \mu \mathrm{m}$ and control $(N=5$ each).

Cells at the radial position of $10 \mu \mathrm{m}$ receive the maximum shear stress of $\sim 2 \mathrm{kPa}$, which is optimum for cell membrane permeabilization. The number of cells transfected was typically $\sim 30$ ( $48 \mathrm{~h}$ after the treatment) for 10 treated cells per sample at $r=10 \mu \mathrm{m}$. We obtain the corrected transfection efficiency of $\sim 75 \%$ as $(30$ transfected cells) divided by $\left(10 \times 2^{n=2}=40\right.$ treated cells) by assuming cells to divide twice $(n=2)$ in the subsequent $48 \mathrm{~h} .{ }^{12,13}$ When the cavitation occurs too close to the cell membrane $(r<5 \mu \mathrm{m})$, the shear stress exceeds $10 \mathrm{kPa}$ and the bubble tends to collapse asymmetrically, often forming high speed liquid jets directed toward the cell membrane, which effectively reduces cell viability. ${ }^{6}$ The control cells were exposed to the CW and nanosecond lasers, with gold nanoparticles in the buffer medium, but without any trapped particle, i.e., no LIB. 
Table 1. Photomechanical transduction process in LIB for water, polystyrene (PS) $400 \mathrm{~nm}$ and gold (Au) $100 \mathrm{~nm}$. Laser pulse energy $E_{p}$, maximum bubble radius $R_{\max }$, mechanical bubble energy $E_{B}$, mechanical transduction efficiency $E_{B} / E_{p}$, and maximum wall shear stress at $r=10 \mu \mathrm{m}$.

\begin{tabular}{cccccc}
\hline & $E_{p}[\mu \mathrm{J}]$ & $R_{\max }[\mu \mathrm{m}]$ & $E_{B}[\mathrm{~nJ}]$ & $E_{B} / E_{p}[\%]$ & $\tau_{w, \max }[\mathrm{kPa}]$ \\
\hline Water $^{4}$ & 8 & 118 & 340 & 4.3 & 1000 \\
PS, 400 nm & 0.58 & 35 & 8.8 & 1.5 & 300 \\
$\mathrm{Au}, 100 \mathrm{~nm}$ & 0.01 & 3 & 0.011 & 0.11 & 2 \\
\hline
\end{tabular}

\subsection{Bubble energy}

LIB of optically trapped microparticles and nanoparticles provided additional degrees of freedom in the domain of the laser-induced cavitation therapy. The energy deposition required for LIB is dependent on the trapped particle material and its size. Table 1 compares the photomechanical transduction process in LIB for water, polystyrene, and gold nanoparticles, respectively, where the energy of a spherical bubble, $E_{B}$, is given by

$$
E_{B}=\frac{4}{3} \pi\left(p_{\infty}-p_{v}\right) R_{\max }^{3}
$$

where $p_{\infty}$ is the static pressure of the surrounding liquid and $p_{v}$ is the vapor pressure of the liquid (2330 $\mathrm{Pa}$ at $293 \mathrm{~K}) .{ }^{14}$ Remarkably, the energy deposition, $E_{p}$, required for the LIB of gold nanoparticles is three orders of magnitude less than that of water. Optical trapping for LIB allows exquisite control of the nanobubble energy, $E_{B}$, from $>300 \mathrm{~nJ}$ (for water) down to $0.01 \mathrm{~nJ}$ (for gold) ranging over four orders of magnitude. The resultant pressure from the cavitation event can be optimized for transfection or delivery of molecules of interest, into a selective zone of cells/tissues or individual cells using this technique of LIB of optically trapped nanoparticles.

In summary, LIB of an optically trapped gold nanoparticle significantly reduces the energy required for cavitation, enabling the single cell transfection and delivery of molecules. This methodology and approach present a significant step forward in the application of optically controlled cell selective transfection.

\section{REFERENCES}

[1] Stevenson, D. J., Gunn-Moore, F. J., Campbell, P., and Dholakia, K., "Single cell optical transfection," J. R. Soc. Interface 7(47), 863-871 (2010).

[2] Mitragotri, S., "Innovation - healing sound: the use of ultrasound in drug delivery and other therapeutic applications," Nat. Rev. Drug Discov. 4(3), 255-260 (2005).

[3] Rau, K. R., Guerra, A., Vogel, A., and Venugopalan, V., "Investigation of laser-induced cell lysis using time-resolved imaging," Appl. Phys. Lett. 84(15), 2940-2942 (2004).

[4] Rau, K. R., Quinto-Su, P. A., Hellman, A. N., and Venugopalan, V., "Pulsed laser microbeam-induced cell lysis: Time-resolved imaging and analysis of hydrodynamic effects," Biophys. J. 91(1), 317-329 (2006).

[5] Arita, Y., Torres-Mapa, M. L., Lee, W. M., Cizmar, T., Campbell, P., Gunn-Moore, F. J., and Dholakia, K., "Spatially optimized gene transfection by laser-induced breakdown of optically trapped nanoparticles," Appl. Phys. Lett. 98(9), 093702 (2011).

[6] Arita, Y., Antkowiak, M., Venugopalan, V., Gunn-Moore, F. J., and Dholakia, K., "Dynamics of primary and secondary microbubbles created by laser-induced breakdown of an optically trapped nanoparticle," Phys. Rev. E 85(1), 016319 (2012).

[7] Antkowiak, M., Arita, Y., Dholakia, K., and Gunn-Moore, F., "Imaging the cellular response to transient shear stress using stroboscopic digital holography," J. Biomed. Opt. 16(12), 120508 (2011).

[8] Arita, Y., Ploschner, M., Antkowiak, M., Gunn-Moore, F. J., and Dholakia, K., "Laser-induced breakdown of an optically trapped gold nanoparticle for single cell transfection," Opt. Lett. 38(17), 3402 (2013).

[9] Novotny, L. and Hecht, B., [Principles of nano-optics], Cambridge University Press, Cambridge (2006).

[10] Seol, Y., Carpenter, A. E., and Perkins, T. T., "Gold nanoparticles: enhanced optical trapping and sensitivity coupled with significant heating," Opt. Lett. 31(16), 2429 (2006). 
[11] Pustovalov, V. K., Smetannikov, A. S., and Zharov, V. P., "Photothermal and accompanied phenomena of selective nanophotothermolysis with gold nanoparticles and laser pulses," Laser Phys. Lett. 5(11), 775-792 (2008).

[12] Tsukakoshi, M., Kurata, S., Nomiya, Y., Ikawa, Y., and Kasuya, T., "A novel method of dna transfection by laser microbeam cell surgery," Appl. Phys. B 35(3), 135 (1984).

[13] Antkowiak, M., Torres-Mapa, M. L., Stevenson, D. J., Dholakia, K., and Gunn-Moore, F. J., "Femtosecond optical transfection of individual mammalian cells," Nat. Protoc. 8, 1216 (2013).

[14] Tomita, Y., Tsubota, M., and An-Naka, N., "Energy evaluation of cavitation bubble generation and shock wave emission by laser focusing in liquid nitrogen," J. Appl. Phys. 93(5), 3039-3048 (2003). 